\section{THREE-MODE: A FORTRAN IV program for three-mode factor analysis}

\author{
JIM MCCLOSKEY \\ Department of Psychology \\ University of Sheffield, Sheffield S10 2TN, England
}

and

\section{PAUL R. JACKSON \\ M.R.C. Social and Applied Psychology Unit University of Sheffield, Sheffield S10 2TN, England}

Data from social science research may frequently be classified and cross-classified in a variety of ways. Traditionally, factor analysis has been defined in terms of two modes of classification, variables and subjects. Its use for multiple classification data beyond two modes typically involves summation methods that ignore the effects beyond the two modes being analyzed. Tucker $(1964,1966)$ has extended the factor-analytic model to allow the investigation of relations in data classified in three or more ways. The Tucker approach not only uses all the sources of variance in data but also specifies the relations between the factors of each of the modes, analogous to the interaction effects found in analysis of variance. One example of a threemode classification of data is the now familiar persons by situations by responses paradigm found in "interactional" psychology. Indeed, researchers in this field have recognized the potential of three-mode factor analysis for investigating the systematic nature of personsituation interaction (Golding, 1975), but they have lacked a suitable computational tool for accomplishing this analysis. The program described here was designed to meet this need, although its range is restricted to data sets defined in two or three modes.

The essence of Tucker's $(1964,1966)$ model is that a data matrix can be described as the product of the factor loading matrices of each mode of classification and the matrix of weights relating the factors of each mode (the core matrix). To date, one of the principal problems in implementing procedures to solve for these matrices has been the difficulty of factoring a very large matrix (as is usually the case for the subjects mode). Tucker (1966) suggested procedures to avoid this problem, but each of them had the drawback that the loadings for the subjects on factors could not be estimated. The advent of virtual storage capabilities on many large computers has alleviated this factoring difficulty to some extent, and the present program takes advantage of this development.

The program follows Method 1, as described by Tucker (1966), but differs in that the calculation of the factor loading matrices is done successively, with the calculation of the approximation to the core matrix intervening each step. One advantage of this program over previous versions (Walsh \& Walsh, 1976) is that a variety of options have been incorporated to allow flexibility in deciding the course of the solution. These options include: (1) specification of missing values in the data; (2) scaling of the data matrix for each mode or combination of the first two modes; (3) type of rotation, varimax or quartimax; (4) specification of the desired number of factors for the solution of each mode; (5) factoring of the three modes without calculating the core matrix, thus saving time in determining the size of the final solution; and (6) a two-mode solution obtainable by specifying only one level for the subjects mode.

Input. The preliminary input defines the size (dimensions) of the data matrix and the type of solution desired (e.g., with or without core, rotation, number of factors, etc.). In general, two runs are required: the first to solve for the roots of the three modes in order to determine the number of factors to be retained for each, and the second to specify the size of the solution and choice of rotation in order to provide interpretable factors and the solution of the core matrix.

The data matrix can be read from the same source as the parameters or from some other input device. The data modes may be in any order of cycling, as the program keeps track of which mode was incremented fastest, and so on. The order of data input is not dependent on the size of the modes; that is, the smallest mode need not be first or the largest mode last.

Output. The initial data specifications and parameters describing the options to be used are output for checking. The scaled data matrix, if requested, is then printed followed by the roots and unit-scaled vectors of each mode. If the number of factors has not been specified, the program decides the number of factors to output for each mode in turn by testing each root against the smallest diagonal element of the sum of squares/cross-product matrix for that mode. This test seems to be reasonably liberal for our own data, in that it has not underestimated the number of factors retained in the final solution. All of the vectors for each mode are also output to a file or card output device to allow easy access in a subsequent run to the vectors for rotation and final calculation of the core matrix, without having to redo the analysis. When the program is being run with the vectors as input, the output consists of the vectors for checking the rotated solution, if requested, and the core matrix. The final item output is always the core matrix.

Computer and Language. The program was written in FORTRAN IV and was developed on an ICL 1906. 
It should be compatible with the FORTRAN compiler of most systems. The subroutine ROTATE published by Cooley and Lohnes (1971) is used.

Core and Time Requirements. The program has 858 statements requiring $12 \mathrm{~K}$ of store with an additional $477 \mathrm{~K}$ for data storage. The test run on a paged computer with a relatively small three-mode matrix ( 12 by 18 by 38 ) required approximately $150 \mathrm{sec}$ to analyze completely (no rotation). This could be improved considerably on a system that is not paged.

Limitations. The program is limited predominantly by the storage capacity of the computer. On our ICL machine, the storage restrictions and program layout have restricted the dimension of the data matrix to 72,500 entries. The maximum number of levels for any one mode is currently set for 220 , but this can be easily changed. If space for a larger data matrix is needed, the set of subroutines that make up the program can be rewritten into separate programs without too much difficulty, thus allowing for the analysis of data matrices involving large numbers of subjects.

Availability. Source listings, reference manual, and a test example may be obtained free of charge from P. R. Jackson, M.R.C. Social and Applied Psychology Unit, University of Sheffield, Sheffield S10 2TN, England. A card deck is available for the cost of postage and packing.

\section{REFERENCES}

COOLEY, W. W., \& LoHNes, P. R. Multivariate procedures for the behavioral sciences. New York: Wiley, 1971.

Golding. S. Flies in the ointment: Methodological problems in the analysis of the percentage of variance due to persons and situations. Psychological Bulletin, 1975, 82, 278-288.

TUCKER, L. R. The extension of factor analysis to three dimensional matrices. In N. Frederickson \& H. Gullikson (Eds.), Contribution to mathematical psychology. New York: Holt, Rhinehart, \& Winston, 1964.

TUCKER, L. R. Some mathematical notes on three mode factor analysis. Psychometrika, 1966, 31, 277-311.

WALSh, J. A., \& Walsh, R. A. A revised FORTRAN IV program for three mode factor analysis. Educational and Psychological Measurement, 1976, 36, 169.

(Accepted for publication October 3, 1978.) 